$\begin{array}{cl}\begin{array}{c}\text { Revue } \\ \text { de /histoire }\end{array} & \text { Revue de l'histoire des religions } \\ \text { des religions } & \begin{array}{l}3 \mid 2015 \\ \text { Varia }\end{array}\end{array}$

\title{
Religion, mystique et crise de la culture à la fin de la période wilhelmienne. Perspectives sur Georg Simmel
}

Religion, mystic and cultural crisis in late Wilhelmian Germany. Perspectives on Georg Simmel

\section{Matthieu Amat}

\section{OpenEdition}

Journals

Édition électronique

URL : http://journals.openedition.org/rhr/8412

DOI : $10.4000 /$ rhr.8412

ISSN : 2105-2573

Éditeur

Armand Colin

\section{Édition imprimée}

Date de publication : 1 septembre 2015

Pagination : 373-396

ISBN : 978-2-200-92993-0

ISSN : 0035-1423

\section{Référence électronique}

Matthieu Amat, «Religion, mystique et crise de la culture à la fin de la période wilhelmienne. Perspectives sur Georg Simmel », Revue de l'histoire des religions [En ligne], 3 | 2015, mis en ligne le 01 septembre 2018, consulté le 01 mai 2019. URL : http://journals.openedition.org/rhr/8412 ; DOI : 10.4000/rhr.8412 


\section{Religion, mystique et crise de la culture à la fin de la période wilhelmienne. Perspectives sur Georg Simmel}

Nous nous proposons d'introduire à la philosophie de la religion de Georg Simmel en tant qu'effort pour dégager la «religiosité» des formes historiques et objectives de la religion. Cette entreprise participant d'une tendance puissante dans la philosophie allemande, au moins depuis Schleiermacher, nous prendrons soin de mettre en avant l'originalité de la réflexion de Simmel. À partir de ses analyses, il nous faudra montrer pourquoi la modernité, comprise comme "crise de la culture», favorise l'essor d'une religiosité de type mystique, et pourquoi elle voit au contraire reculer l'idéal classique de formation de la personnalité par la culture.

\section{Religion, mystic and cultural crisis in late Wilhelmian Germany. Perspectives on Georg Simmel}

We will endeavour to introduce Georg Simmel's philosophy of religion, as an attempt to free "religiosity» from the historical and objective forms of religion. This entreprise partakes of a powerful tendency in German philosophy, at least since Schleiermacher. We will therefore try to highlight the originality of Simmel's reflexion. Using his analysis, we will show why modernity, understood as "a cultural crisis", has fostered a mystical type of religiosity, and why it has, on the contrary, made the classical ideal of personality's formation by culture decline 


\section{VivaCité de L'Élan RELIGIEUX, RECUL DE L'IDÉAL de CULTURE}

Au tournant du siècle et jusque dans les premières années de la république de Weimar, l'Allemagne est le lieu de vifs débats sur l'avenir de la religion et de la culture. Le diagnostic de crise appliqué à l'état de la culture européenne est unanime, qu'on le constate, le déplore ou s'en réjouisse. Les avis divergent quant aux causes et aux remèdes, mais la description est largement partagée: différenciation, voire fragmentation de la culture et absence de vision unitaire du monde; processus d'objectivation liés à l'essor des sciences de la nature, des techniques, et à la monétarisation de l'économie; individualisation et affaiblissement du lien communautaire; relativisation et nivellement des valeurs; crise des religions historiques ${ }^{1}$. Dans ce contexte, la bourgeoisie cultivée doute de l'idéal de formation et de culture qu'elle hérite de l'humanisme allemand et qui l'aide à se définir depuis un siècle².

Contrairement à ce que l'on pourrait croire depuis la France, l'affaiblissement des institutions traditionnelles ne conduit qu'une frange étroite des intellectuels à l'athéisme ou au désintérêt pour la question religieuse. Au contraire, comme le rappelle Marino Pulliero, «le thème d'un réveil religieux devient le refrain de philosophes, théologiens et essayistes $»^{3}$ : prolifération des $«$ religions de substitution»(Ersatzreligionen), engouement pour les religions extra-européennes, essor de la mystique et, d'une manière générale, aspiration à une religiosité libre et intérieure ${ }^{4}$. Celle-ci

1. Pour un état des lieux sur la «crise de la culture» voir Klaus Lichtblau, Kulturkrise und Soziologie um die Jahrhundertwende, Suhrkamp, Frankfurt am Main, 1996 et Aurélien Berlan, La critique culturelle et la constitution de la sociologie allemande. Ferdinand Tönnies, Georg Simmel et Max Weber, ANRT, 2012.

2. Georg Bollenbeck, Bildung und Kultur: Glanz und Elend eines deutschen Deutungsmuster, Frankfurt am Main, Suhrkamp, 1994, III, 2.

3. Marino Pulliero, Une modernité explosive. La revue Die Tat dans les renouveaux religieux, culturels et politiques de l'Allemagne d'avant 1914-1918, Genève, Labor et Fides, 2008, p. 4. Sur ce «réveil religieux», l'ouvrage dresse un panorama impressionnant. Il faut se reporter également à Thomas Nipperdey, Religion im Umbruch: Deutschland 1870-1918, München, Beck, 1988.

4. Le Congrès mondial pour le libre christianisme et le progrès religieux de Berlin, en 1910, constitue un point d'orgue des débats sur la «libre religion». 
ne rompt pas toujours tout lien avec la tradition. Ernst Troeltsch défend par exemple un «libre christianisme» orienté vers un idéal personnaliste, arguant de la continuité entre christianisme et culture moderne (via notamment l'idéalisme kantien)5. Mais l'effort de synthèse du protestantisme culturel est recouvert par les formes de religiosité extra-ecclésiale les plus hétéroclites, du néo-idéalisme d'Eucken au panthéisme moniste, en passant par les options diverses et changeantes des nietzschéens de la Tat, tandis qu'Eugen Diederich satisfait un vaste public en publiant toutes sortes de témoignages sur les religions orientales ou nordiques en même temps que les œuvres de Maître Eckhart". Un «besoin de "religiosité"» se manifeste de toute part, qui mène à une «religiosité vagabondante» plutôt qu'à de la religion au sens strict ${ }^{7}$. Dans ce qui s'apparente à un grand marché, cette «religiosité» recouvre les aspirations les plus diverses et perd en compréhension ce qu'elle gagne en extension. Georg Simmel a tenté de rendre compte philosophiquement de ce phénomène et de proposer un concept consistant de religiosité.

Entre 1898 et 1918, il écrit sept essais consacrés explicitement à la religion. La perspective est tantôt sociologique, tantôt transcendantale, tantôt orientée sur la vie et l'accomplissement religieux, sur fond d'analyse de la modernités. Nous nous concentrerons sur les

Y participèrent des théologiens libéraux (Ernst Troeltsch, Wilhelm Bousset, Otto Baumgarten...) mais aussi beaucoup de non-chrétiens, juifs et bouddhistes en particulier. Voir Pulliero, op. cit. p. 315-326.

5. «Les possibilités d'avenir du christianisme en rapport avec la philosophie moderne», in Ernst Troeltsch, Histoire des religions et destin de la théologie. Oeuvres III (textes traduits et présentés par Jean-Marc Tetaz), Paris-Genève, Cerf-Labor et Fides, 1996. Le Kulturprotestantismus de Troeltsch «fait valoir, comme l'écrit Jean-Marc Tétaz, un personnalisme idéaliste orienté sur l'idéal de culture» (Ibid, p. 522). Sur ce sujet, voir Friedrich Wilhelm Graf, «Rettung der Persönlichkeit. Protestantische Theologie als Kulturwissenschaft des Christentums », in Rüdiger vom Bruch, Friedrich Wilhelm Graf und Gangolf Hübinger (éd.), Kultur und Kulturwissenschaften um 1900, Stuttgart, Steiner Verlag Wiesbaden, 1989.

6. Voir T. Nipperdey, op. cit, p. 143-153.

7. Ibid., p. 143.

8. Zur Soziologie der Religion (1898) et la première édition de Die Religion (1906) participent de la première approche. De la seconde relèvent Beiträge zur Erkenntnistheorie der Religion (BER) (1901), et le deuxième chapitre de Lebensanschauung (LA) (1918). On classera plutôt dans la troisième catégorie Vom Heil der Seele (1902), Die Gegensätze des Lebens und die Religion (GLR) (1904), Ein Problem der Religionsphilosophie (PRP) (1905), la seconde édition de Die Religion (R) (1912) et surtout Das Problem der religiösen Lage (PRL) (1911). Anne-Sophie Lamine propose une classification différente des textes dans «Petite 
textes relevant de cette dernière catégorie, qu'il faudra compléter par les essais de philosophie de la culture, à commencer par la Philosophie de l'argent 9 . En 1918, dans Le Conflit de la culture moderne, Simmel écrit:

[L']impossibilité de préserver plus longtemps les religions transmises par les Églises, tandis que l'élan religieux survit opiniâtrement aux «Lumières» de toute sorte (...) participe des plus profondes difficultés pour d'innombrables hommes d'aujourd'hui ${ }^{10}$.

La situation est différente en ce qui concerne l'idée de culture:

Si aujourd'hui on demandait aux gens des classes instruites quelle idée guide proprement leur vie, la plupart donneraient une réponse spécialisée tirée de leur métier; mais on entendrait rarement parler d'une idée de la culture, concernant l'homme entier et qui dominerait toutes les activités particulières ${ }^{11}$.

Religion et idéal de culture sont dans une situation critique: la foi ne peut plus reposer sur les religions historiques et le métier remplace la culture. Il y a toutefois une dissymétrie frappante. Si la crise que connaissent les Églises est cause d'«innombrables difficultés», l'élan religieux n'est pas mis en question par ces difficultés. En revanche, on assiste à un oubli de l' «idée de culture», en tant que formation de «l'homme entier», par opposition à une

concordance des principaux textes de Georg Simmel sur la religion», Archives des sciences sociales des religions (ASSR), $\mathrm{n}^{\circ}$ 167, 2014, p. 81-82. Seules les analyses sociologiques ont été un peu étudiées en France (Jean Séguy, «Aux enfances de la sociologie des religions: Georg Simmel», ASSR, n 17, 1964, p. 5-11 et Patrick Watier, «Georg Simmel: religion, sociologie et sociologie de la religion», Ibid., $\mathrm{n}^{\circ}$ 93, 1996, p. 23-50). Pas un article des trois volumes des Critical Assessments (David Frisby (éd.), Georg Simmel: Critical Assessments, 3 vol., Londres, Routledge, 1994) ne concerne la religiosité et l'accomplissement religieux. En Allemagne, la littérature n'est pas très abondante, mais un ouvrage est incontournable: Volkhardt Krech, Georg Simmels Religionstheorie, Tübingen, Mohr-Siebeck, 1998. Voir, du même auteur, le riche article: «La religiosité comme seuil. Pertinence contemporaine de la théorie de la religion de Simmel», ASSR, ${ }^{\circ} 167,2014$, p. 61-80 (traduit en français par Violaine Mézière et AnneSophie Lamine).

9. Le concept et la tragédie de la culture (1911), La crise de la culture (1916) et Le conflit de la culture moderne (1918), mais aussi, avant cela, la Philosophie de l'argent (1910). Voir références infra.

10. «Der Konflikt der modernen Kultur» (KMK), in Simmel, GSG 16, p. 205. Traduction en français de Jean-Louis Vieillard-Baron: «Le Conflit de la culture moderne», in Simmel, Philosophie de la modernité, Paris, Payot et Rivages, 2004 (désormais PM). Voir ici p. 403 (trad. modifiée).

11. Ibid., p. 190 (p. 389). Nous indiquerons entre parenthèses la pagination dans la traduction française lorsqu'il y a lieu. 
formation spécialisée. Cette idée n'est plus une référence pour des «classes instruites» qui cessent de s'orienter à partir d'elle. Comment l'expliquer?

Il faut d'abord considérer la manière dont Simmel définit culture et religion en tant que modèles d'accomplissement individuel. Voici ce qu'il écrit dans La crise de la culture, en 1916: «Je comprends [la culture] comme cet accomplissement de l'âme qu'elle n'atteint pas par elle-même, sans médiation, comme c'est le cas dans son approfondissement religieux (...) mais en faisant le détour par les formations du travail spirituel et historique de l'espèce $»^{12}$. Le processus par lequel l'individu se cultive est défini par contraste, et même par opposition, avec un autre idéal d'accomplissement de soi, l'approfondissement religieux. Celui-ci est immanent, celui-là se fait au moyen de produits de l'histoire (sciences, arts, etc.). Pour reprendre les mots de l'essai Le Concept et la Tragédie de la culture, le premier est «uniquement orient[é] vers le sujet», le second désigne la «synthèse d'une évolution subjective et d'une valeur spirituelle objective $»^{13}$. La leçon est double. Tout d'abord l'accomplissement religieux et l'accomplissement par la culture sont incommensurables, voire antinomiques. Ensuite, l'accomplissement religieux est indépendant de la religion en tant que réalité historique (autorité ecclésiale, dogmes, traditions, textes...) et même en tant que fait communautaire.

Quelle consistance peut bien avoir cet «accomplissement»ou «approfondissement» qui semble n'avoir d'autre norme que la subjectivité elle-même? Simmel annonce-t-il les formes les plus individualistes de la religiosité moderne, celles qui conduiront Franz Xaver Kaufmann à parler d' «auto-révérence» ou de «sacralisation du moi $»^{14}$ ? Simmel n'est certes pas le premier à chercher dans la subjectivité l'essence du religieux. Depuis Hegel au moins, c'est

12. «Die Krisis der Kultur», in Simmel, GSG 16, p. 37. Traduction en français de J.-L. Vieillard-Baron: «La crise de la culture», in Simmel, PM. Voir ici p. 411.

13. GSG 14, p. 399 (p. 195-196).

14. Franz Xaver Kaufmann, «Selbstreferenz oder Selbstreverenz? Die soziale und religiöse Ambivalenz von Individualisierung», Ehrenpromotion Franz-Xaver Kaufmann: eine Dokumentation, Bochum, Ruhr-Univ. Pressestelle, 1993 (http:// pub.uni-bielefeld.de/publication/1775037). Voir aussi Hubert Knoblauch, «Die Verflüchtigung der Religion ins Religiöse», in Thomas Luckmann, Die unsischtbare Religion, Francfort a. M., 1991. Sur le rapport de la sociologie des religions (essentiellement allemande) à Simmel, voir V. Krech, op. cit., p. 252-275. 
même plutôt un lieu commun de la philosophie allemande. De ce point de vue, il ne ferait que continuer, et peut-être accentuer, un mouvement dont l'impulsion est attribuée à Luther: l'élimination de «toute condition d'extériorité» pour penser la foi ${ }^{15}$.

Nous montrerons que la position de Simmel doit moins à un Luther hégélianisé qu'à Schleiermacher et la tradition mystique. Nous verrons en outre que la crise de la culture et des religions historiques est l'occasion d'un retour à l'essence de la religiosité. Celle-ci ne nous apparaîtra pas seulement comme un repli subjectif, un refuge dans un contexte de crise, mais comme une forme de vie éminemment moderne - au contraire de l'idéal de culture qui, pour les raisons que l'on verra, dissone dans le contexte moderne.

\section{LA Religiosité SELON Simmel}

\section{Le problème de la situation religieuse}

L'homme du temps présent, qui ne dépend pas intérieurement d'une religion existante, et qui ne considère pas, avec la superficialité des Lumières, le fait des religions comme un simple rêve de l'humanité, dont elle se réveille peu à peu, se trouve à l'égard de ce fait dans une situation d'une indicible inquiétude ${ }^{16}$.

La question de la foi est parfois posée dans les termes d'une regrettable alternative: il s'agirait soit d'accomplir un saut dans la foi et d'accepter l'un ou l'autre des dogmes propres à une religion ou confession historique, soit de s'en tenir à la raison et de suspendre sa croyance - et, éventuellement, faire la critique des contenus objectifs de la religion. L'inquiétude de «l'homme du temps présent » vient de ce qu'il reconnaît la pertinence rationnelle de la critique philosophique de la religion mais constate pourtant que des «esprits de rang supérieur» ont cru et continuent de croire ${ }^{17}$.

15. Selon l'expression hégélienne des Leçons sur la philosophie de l'histoire (trad. Jean Gibelin) Paris, Vrin, 1937, p. 201. On consultera avec profit la sélection de textes proposée par Philippe Büttgen en annexe de Luther, De la liberté du chrétien. Préfaces à la Bible (introduction et traduction par Ph. Büttgen), Paris, Seuil, 1996, p. 149-159.

16. PRL, GSG 14, p. 367. Traduction en français de J.-L. Vieillard-Baron: «Le problème de la situation religieuse», in Simmel, $P M$, p. 339. Après la référence dans le texte allemand, nous indiquons entre parenthèses la pagination

17. Ibid, p. 369 (p. 341, trad. modifiée). 
Il pourrait considérer cela comme une simple curiosité ou l'effet d'une idiosyncrasie, s'il ne ressentait en lui-même «des besoins » sur lequel la critique n'a aucun effet:

[Ainsi] se montre pour la première fois toute la problématique de la situation dans laquelle se trouve aujourd'hui une partie énorme de l'humanité cultivée: à savoir qu'elle est menacée par la puissance renouvelée des besoins dont elle reconnaît comme un pur et simple fantasme les satisfactions offertes jusque-là et actuellement seulement disponibles - et avec lesquelles elle se trouve ainsi complètement dans le vide ${ }^{18}$.

Il n'est plus possible, sauf à s'aveugler, de rejeter la critique des religions historiques. Mais les progrès de la critique, en s'attaquant aux contenus objectifs et historiques des religions, ne font pas disparaître en même temps les besoins auxquels ils répondent. «L'humanité cultivée» ne peut plus croire à ce qu'elle a besoin de croire. Les tentatives pour sortir du dilemme prennent généralement, selon Simmel, une forme pragmatique. On admet que le contenu de foi est «une représentation», un fait subjectif ne renvoyant pas à des réalités objectives - mais on légitime cette représentation par ses effets sur l'individu ou la société ${ }^{19}$. Sous sa forme «mystico-romantique», ce type d'argumentation se retrouve dans des «cercles contemporains d'une haute tenue intellectuelle»: Dieu, Jésus, l'immortalité seraient des «symboles» dont la valeur est indépendante de la question de leur réalité objective. Ces symboles doivent être défendus et conservés, parce qu'ils rattachent à la tradition, parce qu'ils élèvent sentimentalement ou parce qu'ils favorisent un lien communautaire ${ }^{20}$.

Une telle légitimation des contenus de la foi n'est pourtant pas satisfaisante. Une foi qui se comprendrait de la sorte serait-elle d'ailleurs une foi ? Comme l'ajoute Simmel, «la réalité des faits

18. Ibid.

19. Ibid., p. 367 (p. 339).

20. Ibid., p. 368 (p. 340). Simmel pense peut-être ici à Samuel Lublinski, qui attribue à la religion un rôle de synthèse culturelle grâce notamment à ses symboles («Der Organisationsgedanke der Religion», Die Tat 3 (9), 1911, p. 411419), et sans doute à Troeltsch qui, dans «La signification de l'historicité de Jésus pour la foi », présente Jésus comme une «personnalité vivante», «un centre et un symbole» nécessaires à la communauté des croyants (Troeltsch, op. cit., p. 326). Sur le rapport de Troeltsch et Simmel, voir Friedemann Voigt, " "Die Tragödie des Reiches Gottes"? Ernst Troeltsch als Leser Georg Simmels », Troeltsch Studien, Bd. 10, Gütersloher Verlag, München, 1998. 
concernant le salut ne tolère aucun compromis (...), l'élément décisif [est] la réalité absolue de l'être transcendant $»^{21}$. L'argument pragmatique peut satisfaire le psychologue ou le sociologue, l'éducateur ou l'homme politique. Mais il n'est pas recevable en première personne, par celui qui, pour employer une expression un peu désuète, se découvre une aspiration métaphysique. Néanmoins, comme la critique a rendu impossible la satisfaction de cette aspiration par la position d'un objet transcendant, chercher à faire le tri entre ce que l'on peut conserver et ce que l'on doit rejeter dans le dogme est voué à l'échec. Il s'agit donc de savoir s'il peut y avoir un «troisième terme» libérant de cette alternative: soit la foi a un objet transcendant, soit elle n'est rien de plus qu'un fait subjectif. Simmel fait l'hypothèse suivante: "peut-être cette croyance, cet état de fait psychique (seelisch), est-il lui-même quelque chose de métaphysique! $\gg^{22}$. Il s'agit, comme nous allons le voir, de déplacer la perspective de l'objet vers le sujet, de faire du sujet lui-même (plus précisément de sa foi) le lieu essentiel du religieux, sans tomber pour autant dans un réductionnisme psychologique ou sentimental.

\section{De l'objet vers le sujet, de la forme à la vie}

Le texte de 1911 se place sous le patronage de Kant. Celui-ci aurait le premier permis d'appréhender la religion comme «un comportement intérieur de l'âme», une réalité subjective plutôt qu'objective et historique ${ }^{23}$. L'objet, le contenu de la foi, deviennent des produits secondaires et contingents d'une «religiosité» première ${ }^{24}$. L'intention n'est pas d'établir une genèse psychique des phénomènes religieux, mais de considérer l'a priori qui confère à la religion et surtout à la vie religieuse sa valeur spécifique ${ }^{25}$. De ce point de vue, Simmel participe au projet néokantien d'extension de la critique kantienne aux produits

21. Ibid.

22. Ibid., p. 373 (p. 344, trad. modifiée).

23. Ibid., p. 370 (p. 341 ).

24. Ibid.

25. L'anthropologie britannique cherche par exemple à établir la genèse des faits religieux à partir du sentiment de peur. Voir par exemple Edward Tylor, Primitive Culture, Researches into the Development of Mythology, Philosophy, Religion, Language, Art, and Custom, Londres, H. Murray, 1903, vol. 2, p. 209. 
de la culture ${ }^{26}$. Il faut en ce lieu faire un pas en arrière, vers les Contributions à une théorie de la connaissance de la religion de 1901.

Les Contributions insistent sur la distinction entre les contenus historiques de la religion et la «religiosité $»^{27}$. Celle-ci serait l'une des «catégories formelles fondamentales» qui, «à côté d'autres formes fondamentales a priori de notre être intérieur, est capable de faire de toute la richesse de la réalité son contenu », c'est-à-dire de le mettre en forme et de lui donner objectivité et validitée 28 . S'il y a des religions, c'est parce qu'il y a une religiosité capable en droit de donner à tout matériau une coloration spécifique. Le raisonnement est transcendantal: le fait étant donné, il s'agit de remonter à ses conditions de possibilité et de validité. Toutefois, il apparaît rapidement que ce qui intéresse Simmel n'est pas la validité de la religion en tant que fait culturel.

De même que nous devons distinguer le monde objectif qui constitue le contenu du processus de la pensée de ce processus luimême, de même nous devons distinguer le contenu religieux dans sa subsistance objective et sa validité, de la religion en tant que processus subjectif-humain $^{29}$.

Selon une perspective néo-kantienne stricte, cette distinction n'aurait pas lieu d'être, ou ne désignerait que les deux faces d'un même processus, la loi et le fait, l'a priori fondant la légalité, et le fait culturel manifestant cette légalités ${ }^{30}$. Mais Simmel s'intéresse moins à la religiosité comme «catégorie formelle» que comme «processus». À côté de la question de la validité des contenus religieux se pose celle de la valeur de la religiosité elle-même.

Le déplacement du contenu vers la forme conduit à une conception formelle et non substantielle de la religion. Mais cette forme est vivante, elle est, comme Simmel le dira bientôt, une

26. Voir par ex. «Das Heilige», in Wilhelm Windelband, Präludien, Bd. 3, Tübingen, J. C. B. Mohr, 1911 et Heinrich Rickert, System der Philosophie. Teil 1: Allgemeine Grundlegung der Philosophie, Tübingen, J. C. B. Mohr, 1921.

27. BER, GSG 7, p. 14.

28. Ibid.

29. Ibid., p. 10.

30. Là-dessus voir Paul Natorp, «Kant et l'école de Marbourg» dans Cassirer, Cohen, Natorp, L'école de Marbourg (textes traduits par Christian Berner, Fabien Capeillères, Marc de Launay, Carole Prompsy et Isabelle Thomas-Fogiel), Cerf, Paris, 1998, p. 42-43. 
fonction ${ }^{31}$. Si l'on peut utiliser dans une certaine mesure le lexique kantien de l'a priori, il faut prendre soin de penser la forme comme un processus doté d'une «unité intérieure sui generis $»^{32}$. Simmel précise: «l'être le plus intime de la foi religieuse ne me semble pas exprimable autrement: il signifie un état de l'âme humaine, un état de chose réel (Tatsächlichkeit) $»^{33}$. Un «état de chose réel» et non une forme vide, ni un fait psychique descriptible en tant que corrélat ou reflet d'un objet extérieur, réel ou supposé. Ce ne sont pas les contenus de la foi qui distinguent croyants et non croyants, mais l'état de leur âme. La foi en Dieu est ainsi «une espèce de l'existence intérieure $»^{34}$. La foi peut d'ailleurs être en relation avec de tout autres objets: la patrie, la tradition, des idées, etc., et même exister de manière immanente, sans relation à quelque objet que ce soit. Simmel n'est pas clair sur ce point en 1901, mais ce sera net dans Le Problème de la situation religieuse: les contenus ne sont que l'expression et l'objectivation d'une religiosité première ${ }^{35}$. Ainsi, l'âme «n'a pas la religion comme une possession ou un pouvoir, mais au contraire son être est un être religieux $»^{36}$. Il faut donc se garder et du psychologisme et d'une conception intellectualiste de l'a priori, qui manque la dimension vivante de la religiosité ${ }^{37}$.

On ne peut donc en rester à une approche kantienne. Le moment idéaliste (kantien) en philosophie, "par lequel le monde devint "ma représentation"», doit être compensé par une autre attitude

31. «Fonction» ne dénote pas une utilité psychique ou sociale, mais s'oppose à «substance». Est fonctionnel ce qui peut être décrit en termes de relations et d'actions réciproques.

32. Ibid., p. 11.

33. Ibid., p. 17.

34. Ibid.

35. Chez certaines «personnalités géniales», «la religiosité intérieure était si forte (...) que l'être religieux ne pouvait pas soutenir seul sa plénitude (...) mais se projetait dans l'infini pour se reprendre en sens inverse.» (Ibid. p. 382 (p. 352)). Feuerbach a approché cela, mais en réduisant la religion à l'anthropologie il a proposé une lecture psychologique du processus et a cru liquider la transcendance au lieu de la reconnaître dans le sujet lui-même. «Si l'homme pose en face de lui une création métaphysico-divine dépassant toute particularité empirique, ce ne sont pas toujours ni seulement ses émotions psychologiques (...) qu'il projette hors de lui : il projette aussi ce qui est justement métaphysique en lui-même, ce qui se trouve en lui au-delà de toute particularité empirique» (Ibid., p. 374 (p. 345)).

36. PRL, GSG 14, p. 371 (p. 342).

37. Ibid., p. 13. 
philosophique, «un tournant de principe dans la conception du monde»: «objectiver le sujet lui-même $»^{38}$. Il s'agit de rendre sa vie au sujet transcendantal, et non de le considérer comme une simple condition de possibilité des représentations. On peut voir là un retour à un certain réalisme métaphysique - celui d'une métaphysique de la vie, de la relation, et non de la substance. Si le monde est bien «déterminé par la structure d'un sujet» (c'est la position qui est au principe de l'idéalisme philosophique), il faut ajouter réciproquement que «l'acte de représenter est luimême un être qui porte en lui son objectivité et n'a pas besoin de l'emprunter comme un reflet qui lui serait extérieur. C'est le point de vue que nous devons aussi conquérir pour la vie religieuse $»^{39}$. La vie religieuse n'est donc pas une forme en attente de contenu mais « une forme de la vie entière et vivante, une façon dont elle accomplit ses oscillations $\gg^{40}$. La philosophie de la religion a cette forme pour objet: «il s'agit de saisir et de valoriser comme un fait objectif cette religiosité qui naît et persiste dans le sujet, de la reconnaître à sa place dans le monde métaphysique $»^{41}$. Il faut d'une part admettre jusqu'à un certain degré une position réaliste (mais non substantielle), d'autre part reconnaître une valeur spécifique à certains modes d'êtres de l'âme ${ }^{42}$.

La thèse n'est peut-être pas aussi radicale qu'il n'y paraît. «Ma foi est mon être»: c'était déjà, selon Feuerbach, la grande

38. Ibid., p. 378 (p. 349). C'est «une tendance en laquelle se meut déjà la vie intellectuelle de Goethe» (ibid.). Sur la nécessité de compléter le «retour à Kant» et corriger son intellectualisme par un «retour à Goethe», voir «Kant und Goethe», GSG 10, p. 127.

39. Ibid., p. 379 (p. 349, trad. modifiée).

40. Ibid., p. 376 (p. 347).

41. PRL, GSG 14, p. 379 (p. 349).

42. Selon Paul Tillich, Simmel accomplirait le projet schellingien de «psychologie métaphysique», en conférant une objectivité aux «relations constituant la conscience propre à l'expérience de l'absolu». Il procéderait ainsi à une psychologisation non psychologiste du dogme («Religion und Kultur. Die Stellung der Religion im Geistesleben», in P. Tillich, Berliner Vorlesungen, I, Berlin, De Gruyter, 2001, 322-3). P. Tillich loue Simmel pour avoir accompli «la percée la plus décisive vers la désobjectivation (Entgegenständlichung) du concept de religion» (Ibid., p. 439). Dans sa recension de Die Religion en 1906, Troeltsch remarquait déjà que, malgré son apparent psychologisme, Simmel cherchait dans «la pure oscillation de la fonction [religieuse] en elle-même», le «moment du valide et de l'inconditionné» (Troeltsch, Kritische Gesamtausgabe, Walter de Gruyter, Berlin, Bd. 4, p. 525). 
affirmation de Luther, le pendant du «je pense, je suis » cartésien ${ }^{43}$. Simmel proposerait une version «vitaliste» (au sens où elle semble s'inscrire dans la Lebensphilosophie) de ce thème devenu patrimoine de la philosophie allemande. L'enjeu ne serait plus «la certitude qu'a l'esprit de son objectivité »4 ${ }^{44}$ mais une forme de vie et d'accomplissement individuels. Mais l'analogie ne peut être poussée plus loin. Le mouvement de l'objectivité vers la subjectivité n'est pas ici susceptible d'une lecture dialectique. Aussi ne situerat-on pas au mieux la philosophie de la religion de Simmel en lui cherchant une filiation hégélienne. C'est vers une autre tendance de la philosophie post-kantienne qu'il faut se tourner: celle de Schleiermacher.

\section{Religiosité, sentiment et vie. Le rapport à Schleiermacher}

L'année de la parution du Problème de la situation religieuse, Dilthey affirme dans un texte inachevé, Le problème de la religion, que Schleiermacher fut le premier à faire de l'intériorité, «l'instance dernière de la vérité religieuse», en réaction à la critique des dogmes et des traditions menée au dix-huitième siècle ${ }^{45}$. Il faut, selon Dilthey, continuer le travail de Schleiermacher, approfondir la compréhension de «l'expérience religieuse» et montrer qu'elle repose sur une «constitution fondamentale de l'âme» et non sur de

43. Cité par Ph. Büttgen, op. cit., p. 156 (Ludwig Feuerbach, Geschichte der neueren Philosophie von Bacon von Verulam bis Benedikt Spinoza, Sämtliche Werke, Stuttgart-Bad Cannstatt, Fromann-Holzboog, Bd. 3, 1959, p. 15).

44. Ibid.

45. Cité par Gunter Scholtz, «Diltheys "Problem der Religion" », in Archiv für Kulturgeschichte, Bd. 93, Heft 2, 2011, p. 263. Voir dans le même article les pages 263 à 268. Sur les équivoques liées au concept d'intériorité dans la philosophie allemande de la religion, voir $\mathrm{Ph}$. Büttgen, Luther et la philosophie, Paris, Vrin/EHESS, 2009, p. 247-249, ainsi que «L'envers de l'intériorité. Martin Luther », in Gwenaëlle Aubry et Frédérique Ildefonse (dir.), Études sur le moi et l'intériorité, Paris, Vrin, 2008, p. 333-348, et surtout «Unsichtbare Grenzen. Noch einmal zum reformatorischen Gewissensbegriff und dessen Deutung als Signatur der Neuzeit», in Heinrich Neuhaus (dir.), Die Frühe Neuzeit als Epoche, München, Oldenbourg (Historische Zeitschrift, Beiheft 49), 2009, p. 237-250. L'auteur s'en prend à l'équivalence conscience/Temps Modernes, au "pathos de l'intériorité » et au «fétichisme de l'intériorisation » (Verinnerlichung) qui grèvent les lectures de Luther et empêchent de repérer les enjeux pratiques et techniques (pastoraux) du recours par Luther aux termes de Gewissen ou conscientia. 
simples affects ${ }^{46}$. Si c'est un programme schleiermacherien, Simmel s'inscrit dans ce programme. Cela apparaît clairement quand l'on se souvient du point de départ du Problème de la situation religieuse: l'affirmation de la superficialité de la critique des Lumières en ce qui concerne la religion. C'était déjà le geste inaugural des Discours sur la religion, qui s'ouvrent par une exhortation faite aux « hommes cultivés » à distinguer les «sectes et différentes sortes de religion», simples «produit[s] du temps et de l'histoire», et la religion «selon son être intime», «produit de la nature humaine» et non de l'histoire ${ }^{47}$. Cette distinction établie, le lecteur devait reconnaître qu'il n'y a «pas de siècle qui ait réservé un meilleur accueil à la religion que le nôtre $»^{48}$. D'une façon analogue, Simmel tâche de retourner une situation critique en occasion de (re)découverte de la véritable religion.

La proximité entre les textes ne s'arrête pas là. Il est probable en effet que Simmel se souvienne des Discours lorsqu'il en appelle au «tournant de principe dans la conception du monde» qui doit suivre et contrebalancer l'idéalisme. Schleiermacher affirme en effet que la religion ne peut être comprise qu'à partir d'un «réalisme supérieur» qui fasse contrepoids à l'idéalisme ${ }^{49}$. Ce réalisme conduit Schleiermacher à une interprétation métaphysique de la religion. Elle est une «intuition» ou «sentiment de l'infini» produit par l'effet sur l'individu de l'activité de l'univers ${ }^{50}$. Ce sentiment se diffracte en une pluralité de sentiments (amour, respect, humilité, etc.) qui ne sont pas tournés vers l'action ou la connaissance mais valent par eux-mêmes. «Ils sont à éprouver par eux-mêmes, sans autre fin qu'eux-mêmes, en tant que fonctions de [notre] vie la plus intérieure et la plus élevée ${ }^{51}$. L'affirmation de la valeur par soi de la religiosité se retrouve chez Simmel, pour qui le propre de l'accomplissement religieux est $\mathrm{d}^{\prime}$ «éprouver une telle vie comme une valeur absolue $»^{52}$. La religion

46. G. Scholtz, op. cit, p. 267. Wilhelm Dilthey décrit la religiosité comme une «Grundverfassung der Seele » quand Simmel écrit, dix ans plus tôt : «fundamentale Verfassung der Seele» (PRL, GSG 7, p. 12).

47. «Über die Religion», in Friedrich Daniel Ernst Schleiermacher, Kritische Gesamtausgabe, I. Abt. Band 2, p. 198.

48. F.-D.-E. Schleiermacher, Ibid., p. 190.

49. Ibid, p. 213.

50. Ibid. p. 200, 216, et 261.

51. Ibid., p. 214.

52. PRL, GSG 14, p. 380 (p. 350). 
est vie mais libère de la vie en tant que téléologie pratique. À cela s'ajoute encore la proximité lexicale entre les textes simméliens et les Discours (lexique de l'«état d'âme» (Stimmung), de la musique, du sentiment, etc.). Même si Simmel ne parle jamais explicitement des Discours on ne saurait donc douter de leur influence.

C'est sur la question du sentiment religieux que Simmel va tâcher de se distinguer. Il propose à de nombreuses reprises, dans différents textes, des descriptions de la religiosité en termes de sentiments. La religiosité n'est pas «humilité ou élévation, espoir ou contrition, désespoir ou amour, passion ou calme» mais tout cela à la fois ${ }^{53}$. La foi fait «vivre un mélange ou une tension singuliers d'humilité et d'élévation, d'abandon et de désir, de distance et de fusion $»^{54}$. À chaque fois, les sentiments sont associés par couples d'opposés. Or, si l'on en croit un essai de 1905, Un problème de philosophie de la religion, Schleiermacher aurait manqué cette polarité de la religiosité. On sait que dans la Doctrine de la foi ce qui était «sentiment de l'infini» devient «sentiment de dépendance absolue $»^{55}$. Selon Simmel, en «expliqu[ant] la religion à partir du sentiment de dépendance», Schleiermacher serait «unilatéral» : il faudrait également faire droit au «sentiment d'indépendance». La religiosité est «l'être-ensemble de ce sentiment [de dépendance] avec celui d'indépendance $»^{56}$.

Quecette polarité soit absente chez Schleiermacher est discutable ${ }^{57}$. La critique permet en tout cas à Simmel de mettre en avant une position qui prolonge en philosophie de la religion ses intuitions philosophiques fondamentales. Ressentir à la fois la dépendance et l'indépendance: cette apparente contradiction est l'expression d'une structure fondamentale du mode d'être de l'homme. Dans le même texte, Simmel affirme en effet que l'homme a «le double besoin d'appartenir à un plus haut, au plus haut, à un absolu et

53. GLR, GSG 7, p. 296.

54. LA, GSG 16, p. 286. Voir aussi R, GSG 10, p. 51, PRL, GSG 14, p. 377 et BER, GSG 7, p. 13.

55. F.-D.-E. Schleiermacher, Der Christliche Glaube, Berlin, de Gruyter, 1960, Bd. 1, p. 27-28.

56. PRP, GSG 7, p. 319.

57. Denis Thouard a montré que l' «affirmation de la subjectivité finie» dans les Monologues constituait un pendant à l'insistance sur la passivité dans les Discours (D. Thouard, Schleiermacher. Communauté, individualité, communication, Paris, Vrin, 2007, p. 108-109 et 143). Voir également Marianna Simon, La philosophie de la religion dans l'œuvre de Schleiermacher, Paris, Vrin, 1974, p. 120-149. 
en même temps d'être indépendant; d'être accueilli par lui et en même temps de l'accueillir $»^{58}$. Ce motif se retrouve partout chez le philosophe berlinois : dans ses écrits sociologiques (l'individu aspire à s'individualiser et à se socialiser), dans sa philosophie de la culture (l'individu aspire à se former et à se mettre au service de l'esprit objectif), dans sa philosophie de la vie (la vie est auto-limitation et auto-transcendance). D'une manière générale : l'individu est soumis aux exigences de divers touts et aspire lui-même à se constituer comme un tout. De ce point de vue, l'accomplissement religieux apparaît comme une synthèse de ces deux aspirations. C'est cette situation fondamentale de l'homme dans le monde qui se manifeste dans les tensions sentimentales que la religiosité réunit: «humilité ou élévation, espoir ou contrition, désespoir ou amour, passion ou calme (...), le sens de la religiosité est d'offrir un espace commun à tous ces couples de contraires ${ }^{59}$. La religiosité est réconciliatrice et rassemble des tendances contraires dans une unité vivante. Elle est «une tension et une détente de l'âme purement intérieures, un flottement entre une extension et une compression sans bornes de la vie (...) un être-ensemble non interprétable logiquement de puissance et d'impuissance $»^{60}$. Elle est ainsi comparable à un mouvement de diastole et de systole, une pulsation fondamentale par où est expérimenté à la fois l'indépendance de la vie individuelle et son unité avec l'univers ${ }^{61}$.

Loin d'amener à la certitude subjective et réconciliatrice du «chez-soi auprès de soi », l'émancipation de la religiosité vis-à-vis de «toute condition d'extériorité » mène plutôt à l'expérience d'une simultanéité du chez soi et de l'hors de soi. On ne s'étonnera donc pas de voir Simmel trouver ses modèles de vie religieuse non chez un Luther médiatisé par Hegel mais, via Schleiermacher, dans la

58. PRP, GSG 7, p. 319.

59. GLR, GSG 7, p. 296. Sur ce point, voir Hans Joas, Die Enstehung der Werte, p. $116-117$ et V. Krech, art. cit., p. 63 sq.

60. R, GSG 10, p. 69 et 89.

61. Dans ses notes préparatoires à un cours sur la mystique médiévale, Martin Heidegger propose une description semblable de la "piété», à partir de Schleiermacher, mais en critiquant, comme Simmel, l'expression «trop rudimentaire» de «dépendance absolue»: «il faut au contraire interpréter la relation originelle comme l'oscillation entre l'âme et l'esprit absolu et réciproquement» (Heidegger, Phénoménologie de la vie religieuse, Paris, Gallimard, 2012, p. 374). 
tradition de la mystique rhénane ou italienne ${ }^{62}$. Selon Simmel, Luther comprit à raison la foi comme une «constitution (Beschaffenheit) de l'âme», mais il se «figea dogmatiquement». Il ne constitue donc pas la meilleure source pour penser une religiosité indépendante des contenus ${ }^{63}$.

\section{MystiQue, Culture et MOdernité}

\section{Un modèle mystique de religiosité}

Dans sa Georg Simmels Religionstheorie, Volkhardt Krech attire l'attention sur la proximité entre la dialectique simmélienne du tout et de la partie et le motif eckhartien du duplex esse. Selon Eckhart, la créature a un être selon la forme et un être selon la force, elle est formellement individualisée mais absolument dépendante de Dieu. La religiosité simmélienne serait l'expérience unitaire de cet être dual, la réunion de l'être dans la force et de l'être dans la forme, de la dépendance et de l'indépendance ${ }^{64}$.

Si Simmel n'est jamais aussi explicite, on trouve bien des traces de ce thème mystique dans sa description de la religiosité. En outre, dans son cours d'histoire de la philosophie du semestre d'hiver 1913/14, la mystique occupe une place conséquente dans la partie consacrée à la philosophie du Moyen Âge, et Eckhart est l'objet d'une attention particulière: «il est non seulement le premier grand philosophe spéculatif allemand, mais peut-être même, en ce qui concerne la profondeur, l'élan et la force de la pensée spéculative, le

62. Simmel parle peu de Luther, dont le nom n'est mentionné qu'une fois dans les leçons d'histoire de la philosophie (GSG 21), quand celui d'Eckhart l'est une vingtaine de fois et celui de Nicolas de Cuse huit fois. Schleiermacher y est explicitement rapproché de la tradition mystique: par la manière dont il relie l'individuel et l'universel, il serait, comme Nicolas de Cuse, un philosophe de la «coincidentia oppositorum» (Ibid., p. 32). Quelques années plus tard, cette figure d'un Schleiermacher mystique devient un repoussoir pour ce qui deviendra la théologie dialectique: Emil Brunner Die Mystik und das Wort, Tübingen, 1924. La ligne tirée entre la tradition mystique et Schleiermacher passe bien sûr par le piétisme (voir par ex. Michel Cornuz, Le Protestantisme et la mystique: entre répulsion et fascination, Labor et Fides, Genève, 2003, p. 18 sqq. et 73 sqq.).

63. GSG 21, p. 834. Cette critique de Luther est un lieu commun du protestantisme libéral et, avant lui, du spiritualisme piétiste.

64. V. Krech, op. cit., p. 211 sq. V. Krech s'appuie sur Meister Eckhart, Lateinische Werke I (traduit et édité par Konrad Weiss), Stuttgart, 1964 , p. 238. 
plus grand que l'Allemagne n' ait jamais eu $»^{65}$. Il serait en particulier parvenu à formuler ce qui constitue le noyau de la mystique: «s'abîmer absolument dans le moi propre, se détacher de tous les contenus particuliers donnés de l'extérieur, est l'unique chemin permettant de saisir l'absolu $»^{66}$. Cette description subjectiviste du processus mystique suggère que c'est à Eckhart que Simmel emprunte sa description de l'«approfondissement religieux» comme «accomplissement de l'âme (...) sans médiation». La vraie religion est donc non seulement religiosité, mais religiosité mystique $^{67}$.

Il est clair que ce mouvement est contraire à celui de la culture, qui s'effectue, comme nous l'avons dit en introduction, par un «détour par les formations du travail spirituel et historique de l'espèce», et commence donc par une sortie de soi. La culture, précise Simmel dans le fameux essai Le Concept et la tragédie de la culture, «est le chemin qui va de l'unité close à l'unité déployée en passant par le déploiement de la multiplicité ${ }^{68}$. Elle est un mouvement centrifuge d'appropriation de contenus. L' «âme» se cherche d'abord à l'extérieur d'elle-même et se constitue en quelque sorte une périphérie avant que d'avoir trouvé son propre centre - charge à elle de réaliser ensuite la synthèse de sa vie subjective et des contenus objectifs. Aussi la culture forme-t-elle les esprits d'une manière profondément différente de la religion. En 1911, Simmel écrit à Rickert:

Depuis peu je me consacre de nouveau à Maître Eckhart et je suis à nouveau tout à fait impressionné par la profondeur et la liberté de cet esprit. L'Allemagne a produit des esprits plus étendus et plus différenciés; je doute en revanche qu'elle en ait produit un plus profond, plus concentré ${ }^{69}$.

65. GSG 21, p. 29.

66. Ibid.

67. En 1910, lors du premier Deutschen Soziologentag, Simmel soutient, après que Troeltsch a présenté sa partition de la religion en type-église, type-secte et type-mystique, que seule la mystique est authentiquement religion (voir Krech, op. cit., p. 210).

68. BTK, GSG 14, p. 387. Traduction en français de Philippe Ivernel: «Le concept et la tragédie de la culture » in Simmel, La Tragédie de la culture, Paris, Rivages, 1988 (voir ici p. 182).

69. Lettre à H. Rickert du 29 décembre 2011 (GSG 22, p. 1021). Voir aussi la lettre à Martin Buber du 14 novembre 1910, où Simmel loue la philosophie chinoise en la rapprochant de celle de Maître Eckhart, et celle du 16 janvier 1911 à Emil Diederich dans laquelle il exprime son impatience de la parution d'un volume 
Les «esprits plus étendus et plus différenciés » sont les philosophes d'entendement, du type de Kant, qui se caractérisent par leur orientation objective. On ne saurait superposer parfaitement culture et philosophie, mais ce qui vaut ici pour les philosophes vaut pour les esprits cultivés. La différenciation interne est en effet le reflet des intérêts multiples, dirigés vers différentes sphères d'objectivités, intérêts caractérisant précisément la culture. L'orientation religieuse mystique préserve quant à elle d'une extension mal contrôlée et d'une différenciation menaçant de devenir fragmentation. Elle indique une voie de résistance à l'objectivation et à l'égarement dans la multiplicité.

Ce n'est toutefois pas en ce point que la position de Simmel est la plus originale. En appeler à l'âme et à la vie contre l'esprit (intellectuel, objectif, etc.) est un lieu commun de la philosophie de la vie et de la Kulturkritik ${ }^{70}$. L'apport le plus intéressant de Simmel réside dans les conséquences qu'il tire de sa description de la religiosité: dans l'affirmation de l'affinité profonde entre mystique et modernité.

\section{Mystique et « vie moderne»}

La modernité peut être caractérisée par les processus suivants, décrits précisément dans la Philosophie de l'argent: individualisation croissante; dissolution du substantiel dans le fonctionnel; différenciation et éclatement de la culture objective et donc des exigences qui pèsent sur l'individu ${ }^{71}$. La religiosité mystique n'est pas

d'Eckhart. La valorisation de la mystique chez Simmel excède la philosophie de la religion: "la théologie négative de la mystique s'est avancée plus librement et plus profondément que toute la dogmatique ou la philosophie de la religion antérieures ou ultérieures» (LA, GSG 16, p. 306). Selon Margarete Susman, le «fondement le plus profond» de la pensée de Simmel est d'ailleurs la mystique, et non la philosophie de la vie (Die geistige Gestalt Georg Simmels, Tübingen, J. C. B. Mohr, 1959, p. 8).

70. Voir «Réflexion sur le caractère «affirmatif» de la culture», in Herbert Marcuse, Culture et société, Paris, Minuit, 1970, p. 116 sq.

71. La modernité (Moderne) désigne parfois les Temps modernes (Neuzeit) mais plus souvent la période qui leur succède - après la révolution bourgeoise puis industrielle, et avec le tournant kantien dans l'histoire des idées. Des trois processus évoqués, le second est le plus décisif. Voir «Rodin», GSG 14, p. 346 : "L'essence de la modernité (...) est la dissolution des contenus stables dans l'élément liquide de l'âme, à partir de laquelle toute substance est éliminée, et dont les formes ne sont que des formes du mouvement». 
seulement compatible avec ces processus, elle les accompagne; mieux: elle les accomplit.

En 1904, dans Les oppositions de la vie et de la religion, Simmel écrit: "l'homme a besoin de religion pour la réconciliation de la division entre ses besoins et leur satisfaction, entre son devoir et son faire, entre son image idéale du monde et la réalité $\gg^{72}$. Or, ce besoin ne peut désormais plus être satisfait par des formations objectives et institutionnelles, et c'est à l'individu que revient la tâche de la réconciliation. De là le regain d'intérêt pour la mystique: celle-ci fait reposer sur l'âme seule l'entreprise de réconciliation. Aussi Simmel ajoute-t-il : «Toute mystique est un mélange du moi avec la divinité, parce qu'il n'y a pas d' autre voie que notre âme pour amener l'insupportable multiplicité et étrangeté des choses vers l'unité ${ }^{73}$. Cette proposition prend un relief nouveau lorsque les capacités d'intégration des institutions et des discours s'affaiblissent, tandis que croît la différenciation de la culture. De nouvelles prestations sont alors attendues de la religion. «L'homme moderne», n'attend plus d'elle une «image du monde» mais une aide pour affronter les «oppositions intérieures de la vie ${ }^{74}$. Or, considérer la religion de manière objective, à partir de ses contenus dogmatiques, ne fait qu'accentuer les oppositions et ajouter aux exigences objectives qui pèsent sur l'individu. L'orientation subjective de la religiosité indique une voie d'accomplissement qui n'est pas une orientation objective de plus.

La religiosité, même mystique, ne permet certes pas de se libérer de l'ensemble des exigences objectives et parfois opposées (économiques, politiques, éthiques, techniques, scientifiques, etc.) que fait peser la culture moderne sur l'individu. Mais comprise à partir du double mouvement esquissé plus haut, cette religiosité indique un dépassement relatif de ces oppositions. En effet, elle permet de penser la religion comme «un membre et en même temps un organisme entier, une partie de l'existence et en même temps l'existence elle-même à un niveau (Stufe) plus élevé et plus intégré ${ }^{75}$. Selon le point de vue, la religiosité est réconciliatrice ou prend part au combat. Le processus est irréductiblement conflictuel,

72. GLR, GSG 7, p. 302

73. Ibid.

74. Ibid.

75. Ibid., p. 302. 
mais l'on sait que le conflit participe, chez Simmel, à la constitution d'unités supérieures. Dans le contexte moderne, l'accomplissement religieux ne peut être qu'un «développement incessant au travers d'oppositions se présentant toujours à nouveau $»^{76}$. L'unité de la vie (religieuse) n'est qu'une idée régulatrice, une «tâche infinie » ${ }^{77}$. Il n'y a pas d' «instance réconciliatrice» se tenant en un «repos immaculé au-delà de toutes les oppositions $\gg^{78}$.

Parce que la religiosité moderne est mystique, fonctionnelle et individuelle, elle s'accorde au «rythme de la vie moderne», au point qu'elle apparaît à certains égards comme sa forme sublimée ${ }^{79}$. On sait que pour Simmel le symbole par excellence de la modernité est l'argent - la philosophie de la culture moderne est une philosophie de l'argent. Or, celui-ci est sans qualité, fluide, purement fonctionnel, mais peut néanmoins être échangé contre des objets de toute qualité. Il se cristallise ou se fluidifie. Il est un objet parmi d'autres, une partie du tout des objets convertibles en argent, mais il est aussi ce tout lui-même, du moins de manière fonctionnelle. Il est un tout et une partie, lui-même et autre chose que lui-même. Son mouvement l'apparente donc formellement à celui de la vie religieuse. L'analogie est expressément établie dans la Philosophie de l'argent:

L'argent participe (...) des puissances de la vie qui répondent à ce schéma très particulier: (...) elles s'élèvent au-dessus des oppositions dans lesquelles se divise la province d'intérêts concernée, (...) mais c'est pour ensuite, ou en même temps, devenir partie, là où elles venaient d'être ou juges ou impartiales. Ainsi tout d'abord de la religion, dont l'homme a besoin pour apaiser la coupure entre ses besoins et leur satisfaction, entre son devoir et sa praxis (...). Cependant, une fois créée par lui, la religion ne demeure pas sur les sommets atteints dans ses moments les plus sublimes, mais descend elle-même dans l'arène, devient une face de ce dualisme de l'existence qu'elle venait justement de résorber en elle ${ }^{80}$.

L'intérêt de la modernité pour la mystique ne saurait donc être une simple mode. Il s'explique par la similitude fonctionnelle entre les formes de la vie moderne et la description mystique de la vie

76. Ibid.

77. Ibid. Là-dessus voir V. Krech, op. cit., p. 113 sq.

78. Ibid., p. 303.

79. Ibid.

80. GSG 6, p. 692. Traduction en français de Sabine Cornille et Philippe Ivernel: G. Simmel, Philosophie de l'argent, Paris, PUF, 1987 (ici p. 639). 
religieuse de Eckhart à Nicolas de Cuse - ce dernier étant considéré par Simmel, en vertu de ses développements sur la coincidentia oppositorum, comme un «prophète du sentiment de la vie des temps modernes ${ }^{81}$. La coloration mystique de la religiosité moderne n'est pas seulement la réaction à une modernité matérialiste, objectiviste et atomisante ${ }^{82}$. Elle y résiste en effet; mais si l'on comprend la modernité d'abord comme un devenir-fonctionnel, un processus de désubstantialisation, on devra reconnaître que la religiosité mystique l'accomplit plutôt qu'elle ne s'y oppose.

\section{Culture, religion et rejet de la forme}

Le processus individuel de culture n'est en revanche nullement favorisé par le «rythme de la vie moderne». En tant que mouvement excentrique dirigé vers de l'esprit objectivé (œuvres, textes, théories, etc.), il implique de reconnaître des valeurs objectives (esthétiques, scientifiques, etc.). Il suppose d'abord «une satisfaction pour ainsi dire objective à ce que cette œuvre se tienne là-devant», dans son altérité et son autonomie ${ }^{83}$. Tout l'oppose donc à l'accomplissement religieux qui éprouve la vie «comme une valeur absolue». Du point de vue de l'idée de culture, la vie n'est pas une valeur, ou seulement une valeur parmi d'autres. La culture implique en outre que «des formations objectives, sans rien perdre de leur objectivité, se trouvent intégrées dans le processus d'accomplissement des sujets ${ }^{84}$. Même dans le retour de l'objet vers le sujet se maintiennent donc des évaluations objectives. Là encore, ce n'est pas une forme de vie qui est par elle-même éprouvée comme une valeur.

La valorisation de certains contenus objectifs est condition de la culture et ennemie de la religiosité. Lorsque celle-ci se détermine en fonction de formations objectives, elle se transforme précisément en culture. Or, un des caractères de la modernité est précisément l'accroissement formidable de la quantité de contenus objectifs

81. Nicolas de Cuse «a anticipé des sentiments extrêmement modernes, il a d'une certaine manière anticipé notre temps » (GSG 21, p. 31.).

82. Sur cette thèse alors extrêmement répandue, voir par ex. Rudolf Eucken, Geistige Strömungen der Gegenwart, Leipzig, Veit \& Comp., 1904 ou W. Windelband, «Von der Mystik unserer Zeit» (1910), in Präludien, Bd. I, Tübingen, J. C. B. Mohr, 1915.

83. BTK, GSG 14, p. 392 (p. 188, trad. modifiée).

84. Ibid. p. 413 (p. 213, trad. modifiée). 
disponibles (arts, sciences, savoirs, etc.). On pourrait penser que cela favorise la culture individuelle. Il faut pourtant rappeler ce constat, qui constitue un des points de départ de la philosophie simmélienne de la culture:

si nous envisageons la culture énorme qui s'est incarnée depuis cent ans en objets et en connaissances, en institutions et en confort, et si nous lui comparons le progrès de la culture individuelle dans le même temps (...) une différence effrayante de croissance se montre entre les deux, et même en plusieurs points une régression de la culture individuelle ${ }^{85}$.

La dynamique massive d'objectivation des sociétés hautement différenciées a pour effet tantôt de soumettre l'individu à des normes purement objectives, tantôt le paralyse et le rejette dans la sphère subjective, tantôt l'excite de manière superficielle et sans aucun profit: «la réserve d'esprit objectivé croissant à l'infini pose des exigences au sujet, éveille des velléités en lui, l'accable du sentiment de sa propre insuffisance et impuissance $»^{86}$. Ces conditions empêchent la synthèse de subjectif et d'objectif en quoi consiste la culture.

L'autonomisation et l'extension de l'esprit objectivé va par ailleurs de pair avec le processus de dissolution du substantiel dans le fonctionnel. L'évolution des sciences en témoigne d'une manière significative: les questions de méthode occupent une place de plus en plus considérable, l'objectivité ne concerne que des relations mobiles, les éléments des savoirs constituent des séries toujours plus complexes ${ }^{87}$. Les contenus se fragmentent et n'ont plus de sens qu'en fonction des autres, dans un jeu de relations et de renvois infinis ${ }^{88}$. Les contours et les formes s'estompent. Ce mouvement s'étend à toutes les sphères de la culture, de l'économie à l'art, où l'expressionnisme cherche l'expression directe de la vie ${ }^{89}$. Dans $L e$ conflit de la culture moderne, la «culture moderne» est caractérisée par le «rejet du principe même de la forme», c'est-à-dire «l'abandon du classicisme en tant qu'idéal absolu de l'humanité et de la

85. «Die Großstädte und das Geistesleben», GSG 7, p. 129. Traduction en français de J.-L. Vieillard-Baron: «Les Grandes villes et la vie de l'esprit», in Simmel, PM, p. 181.

86. BTK, GSG 14, p. 412 (p. 212).

87. Là-dessus, voir GSG 6, p. 94-95.

88. «Persönliche und sachliche Kultur», GSG 5, p. 573-574.

89. KMK, GSG 16, p. 190-1 (p. 390-391). 
culture $»^{90}$. Or l'idéal de culture individuelle parait irréductiblement classique, dans la mesure où la culture n'est pensable que comme mise en forme, à partir d'objets eux-mêmes dotés d'une forme - d'où le rôle culturel éminent que Simmel assigne, après tant d'autres, aux œuvres d'art ${ }^{91}$. Le processus moderne de dissolution de la substance dans la fonction semble donc mener nécessairement au recul de l'idéal de culture. La mystique est quant à elle le type de religiosité adéquat à une époque qui rejette la forme:

Dans la direction de la mystique, ce qui se montre le plus décisif, c'est qu'ainsi la directivité et la détermination des limites de la forme religieuse sont supprimées (...) ici se trouve une ampleur indéterminée du sentiment religieux qui ne se heurte pas à des bornes dogmatiques, et qui s'approfondit dans une infinité sans forme ${ }^{92}$.

Un certain scepticisme se manifeste toutefois chez Simmel quant à la possibilité d'une telle religiosité sans forme. Dans $L e$ problème de la situation religieuse, Simmel doutait déjà de la possibilité que «la religiosité des hommes moyens accompli[sse] le tournant qui l'éloigne de la substance $»^{93}$. Le modèle mystique d'accomplissement religieux est à certains égards un modèle aristocratique. Il va plus loin dans Le conflit de la culture moderne puisque peu après le passage que nous venons de citer, il affirme que l'idée d'une «mutation de la foi transitive en une foi intransitive», aussi séduisante soit-elle, désigne sans doute un «intermède» dans l'histoire de la religion plutôt qu'un tournant définitif. La vie, même religieuse, ne se réalise toujours «que dans la forme de son adversaire, c'est-à-dire dans une forme $»^{94}$. On peut interpréter cela comme un aveu d'échec quant à la tentative de constituer un concept de religiosité rigoureusement fonctionnel ${ }^{95}$. Cela n'ôte pas leur valeur aux deux principaux résultats de la recherche: la démonstration d'une part de l'affinité profonde entre aspiration mystique et culture moderne, d'autre part du fait que le rejet des formes historiques de la religion n'est pas nécessairement l'expression d'un subjectivisme inconsistant.

90. Ibid. (p. 398).

91. Voir par ex. «Kant, Sechzehn Vorlesungen», GSG 9, p. 68.

92. KMK, GSG 16, p. 202 (p. 400).

93. PRL, GSG 14, p. 383 (p. 353, trad. modifiée).

94. KMK, GSG 16, p. 204-205.

95. Sur le caractère insatisfaisant des définitions fonctionnelles de la religion, voir H. Knoblauch, art. cit., p. 12. 
Quelles que soient les possibilités de son accomplissement, la religiosité moderne participe bien, aux côtés de l'économie capitaliste monétarisée ou du refus de l'œuvre dans l'art, d'un processus de dissolution générale des structures institutionnelles et des formations objectives. Dans cette situation, il n'est pas étonnant que la mise en avant de l'idéal de culture prenne parfois une tonalité conservatrice et «anti-moderne». Ce n'est pas tant le signe de la nostalgie d'une culture, au sens d'un état de fait objectif, que le refus d'abandonner la culture, comme modèle de formation individuelle - et collective. Comme celui-ci n'est possible qu'à la condition qu'une valeur spécifique soit reconnue à des produits historiques stabilisés, il arrive que la rhétorique mobilisée rejoigne celle des autorités religieuses traditionnelles, attachées à leur objectivité institutionnelle et dogmatique ${ }^{96}$.

matthieuamat@gmail.com

96. Ce travail doit beaucoup aux conseils et remarques de Philippe Büttgen, Linda Hennig et Denis Thouard. Qu'ils en soient ici chaleureusement remerciés. 